\title{
Steps or Terraces? Dynamics of Aromatic Hydrocarbons Adsorbed at Vicinal Metal Surfaces
}

\author{
Javier Camarillo-Cisneros, ${ }^{1,3}$ Wei Liu, ${ }^{1,2}$ and Alexandre Tkatchenko, ${ }^{1, *}$ \\ ${ }^{1}$ Fritz-Haber-Institut der Max-Planck-Gesellschaft, Faradayweg 4-6, D-14195 Berlin, Germany \\ ${ }^{2}$ Nano Structural Materials Center, School of Materials Science and Engineering, Nanjing University of Science and Technology, \\ Nanjing 210094, Jiangsu, China \\ ${ }^{3}$ Centro de Investigación en Materiales Avanzados, Miguel de Cervantes 120, C.P. 31109, Chihuahua, Mexico
}

(Received 30 January 2015; published 20 August 2015)

\begin{abstract}
The study of how molecules adsorb, diffuse, interact, and desorb from imperfect surfaces is essential for a complete understanding of elementary surface processes under relevant pressure and temperature conditions. Here we use first-principles calculations to study the adsorption of benzene and naphthalene on a vicinal $\mathrm{Cu}(443)$ surface with the aim to gain insight into the behavior of aromatic hydrocarbons on realistic surfaces at a finite temperature. Upon strong adsorption at step edges at a low temperature, the molecules then migrate from the step to the (111) terraces, where they can freely diffuse parallel to the step edge. This migration happens at temperatures well below the onset of desorption, suggesting a more complex dynamical picture than previously proposed from temperature-programed desorption studies. The increase of the adsorption strength observed in experiments for $\mathrm{Cu}(443)$ when compared to $\mathrm{Cu}(111)$ is explained by a stronger long-range van der Waals attraction between the hydrocarbons and the step edges of the $\mathrm{Cu}(443)$ surface. Our calculations highlight the need for time-resolved experimental studies to fully understand the dynamics of molecular layers on surfaces.
\end{abstract}

DOI: 10.1103/PhysRevLett.115.086101

PACS numbers: 68.43.-h, 68.35.Ja, 68.60.Dv

Microscopic understanding of the thermodynamics and kinetics of organic molecules at inorganic substrates plays an increasingly important role in modern surface science and technology [1-6]. Obviously, the predictive modeling and understanding of the structure, stability, and dynamics of such hybrid systems is an essential prerequisite for tuning their electronic properties and functions [7-10]. In recent years, remarkable progress has been achieved in understanding the structure and stability of molecules on close-packed surfaces by an intensive joint effort between experiment and theory [11]. For some of the well-defined model systems, such as benzene adsorbed on close-packed (111) metal surfaces, state-of-the-art calculations and experiments now often agree to better than $0.1 \AA$ in vertical adsorption heights and $0.1-0.2 \mathrm{eV}$ in adsorption energies [11-13]. However, the functionality of realistic interfaces with applications in catalysis, lightemitting diodes, single-molecule junctions, molecular sensors and switches, and photovoltaics is often determined by interactions of molecules with more complex ("imperfect") substrates [3,14-18]. Therefore, a complete understanding of the dynamics and reactivity of such interfaces demands reliable modeling of the interaction of adsorbed organic molecules with steps, kinks, impurities, and defects. Obviously, the main difficulty here consists of accurately capturing all the relevant energy contributions involved in binding on imperfect substrates, including Pauli repulsion, covalent hybridization, charge transfer, and van der Waals (vdW) attraction. Especially difficult are cases in which both chemical bonds and vdW interactions make substantial contributions to the overall adsorption process.

In this context, this Letter focuses on modeling and understanding the adsorption, diffusion, and desorption (ADD) mechanisms of fundamental aromatic hydrocarbons-benzene $(\mathrm{Bz})$ and naphthalene $(\mathrm{Np})$ - on the vicinal $\mathrm{Cu}(443)$ surface. The temperature-dependent behavior of these systems has been previously studied by temperature-programed desorption (TPD), low-energy electron diffraction (LEED), and scanning tunneling microscopy (STM) [19]. It was concluded that a visible increase in the desorption temperature for $\mathrm{Bz}$ on $\mathrm{Cu}(443)$ compared to $\mathrm{Cu}(111)$ stems from a stronger adsorption along the step edges in the former case. Our calculations provide an alternative three-step mechanism for the dynamics of $\mathrm{Bz}$ and $\mathrm{Np}$ on the vicinal $\mathrm{Cu}(443)$ surface. We find that, upon strong adsorption on step edges at a low temperature, the molecules then migrate from the step to the (111) terraces, where they can freely diffuse parallel to the step edge. This migration happens at temperatures well below the onset of desorption. The increase in the desorption temperature observed in experiments for the $\mathrm{Cu}(443)$ surface when compared to $\mathrm{Cu}(111)$ is explained by a stronger $\mathrm{vdW}$ attraction between the hydrocarbons and the step edges of the $\mathrm{Cu}(443)$ surface.

All calculations in this work employ dispersion-inclusive density-functional theory (DFT), specifically using the DFT $+v d^{\text {surf }}$ method [20], combined with the 
Perdew-Burke-Ernzerhof (PBE) exchange-correlation functional [21]. The PBE $+\mathrm{vdW}^{\text {surf }}$ method has already been successfully applied to study the adsorption and reactions of a wide variety of molecules on (111) metal surfaces, often achieving quantitative accuracy for structures and adsorption energies in comparison to state-of-theart $\mathrm{x}$-ray standing wave, TPD, and microcalorimetry experiments $[11,22,23]$. In this study, the PBE $+v \mathrm{vW}^{\text {surf }}$ method is applied to a vicinal surface for the first time, demonstrating that its relative accuracy also extends to adsorption on stepped substrates. Most calculations in this work were carried out using the all-electron code FHI-aims [24]. Transition-state geometries were determined using the CASteP code $[25,26]$. See Supplemental Material (Ref. [27]) for more details about our DFT calculations and convergence studies. Our calculations are fully converged in terms of numerical settings, using parameters employed before in our study of $\mathrm{Bz}$ adsorption on transition metal surfaces $[12,13]$.

Before proceeding to study the ADD behavior of $\mathrm{Bz}$ and $\mathrm{Np}$ on $\mathrm{Cu}(433)$, we start by systematically analyzing the static potential-energy surface (PES) for these systems. We have carried out extensive geometry optimizations starting from different initial structures, where the $\mathrm{Bz}$ and $\mathrm{Np}$ molecules are situated in different adsorption sites and rotated by either $0^{\circ}$ or $30^{\circ}$ with respect to the [110] direction of the $\mathrm{Cu}(443)$ surface. As can be appreciated in Figs. 1(a) and 1(b), there is a clear preference for adsorption of $\mathrm{Bz}$ and $\mathrm{Np}$ on top of step edges and the appearance of many metastable states near the step edges and on terraces of the $\mathrm{Cu}(443)$ surface. This complex situation is in marked contrast with the close-packed $\mathrm{Cu}(111)$ surface, where the PES for the adsorbed $\mathrm{Bz}$ and $\mathrm{Np}$ is essentially flat and their ADD dynamics are well understood [13]. TPD studies of $\mathrm{Bz} / \mathrm{Cu}(111)$ exhibit a single peak in the desorption spectrum, and STM investigations suggest that the Bz molecules are freely diffusing on the $\mathrm{Cu}(111)$ surface at a low temperature [28]. On the $\mathrm{Cu}(443)$ surface, the preferred adsorption on step edges can be explained by reactive dangling bonds, and therefore both $\mathrm{Bz}$ and $\mathrm{Np}$ are able to form weak chemical bonds with the $\mathrm{Cu}$ atoms there. In fact, the most stable step-edge adsorption sites for both $\mathrm{Bz}$ and Np exhibit adsorption energies that are larger by $\sim 0.45 \mathrm{eV}$ for $\mathrm{Bz}$ and $\sim 0.60 \mathrm{eV}$ for $\mathrm{Np}$ compared to the binding strength on the close-packed $\mathrm{Cu}(111)$ surface. The adsorption energies of $\mathrm{Bz}$ and $\mathrm{Np}$ and the structures of $\mathrm{Bz}$ on $\mathrm{Cu}(443)$ for several characteristic configurations found in our extensive exploration of the PES are shown in Figs. 1(a)-1(c). The symmetry breaking introduced by step edges leads to a range of adsorption energies on the (a)

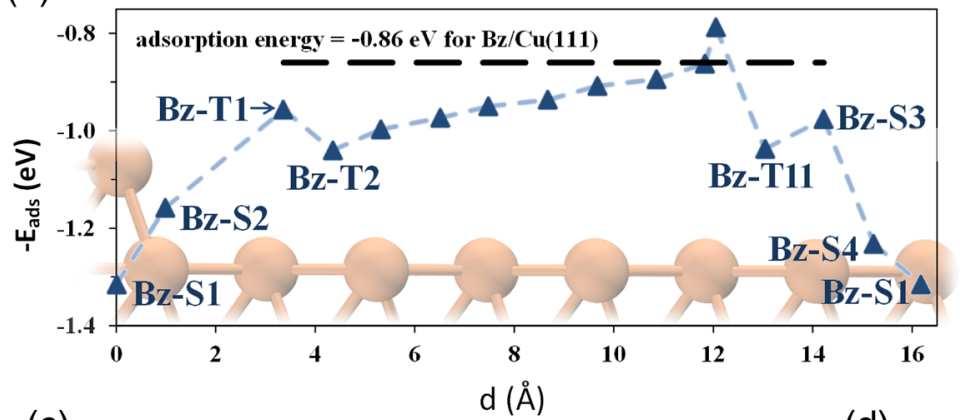

(c)

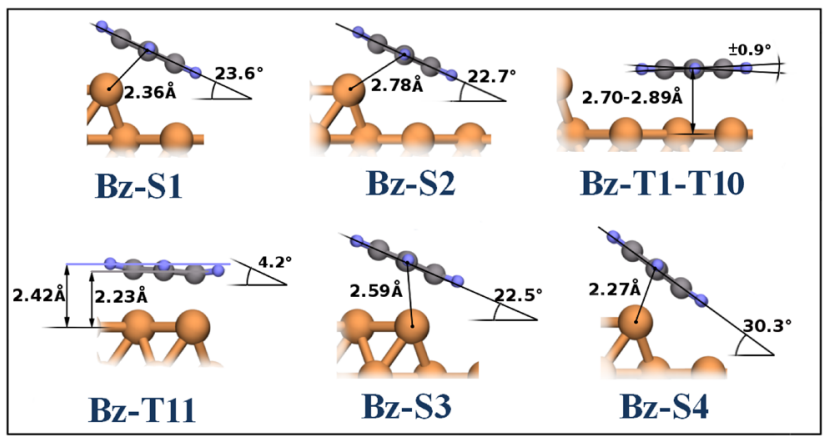

(b)

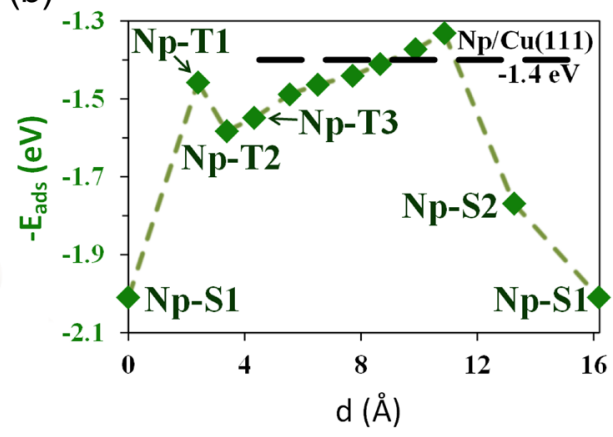

(d)

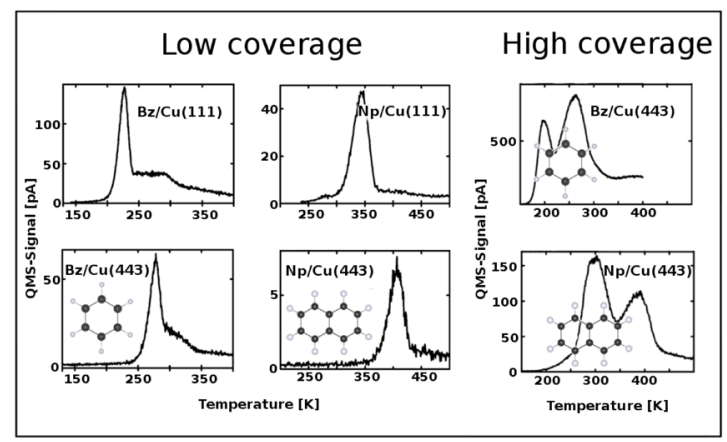

FIG. 1 (color online). Adsorption energy as a function of distance $d$ measured from the molecule center of mass to the step edge of the $\mathrm{Cu}(443)$ surface. Geometries and energies were obtained using the $\mathrm{PBE}+\mathrm{vdW}^{\text {surf }}$ method for (a) benzene/Cu(443) and (b) naphthalene/ $\mathrm{Cu}(443)$. The letters " $S$ " and " $T$ " denote the "step" and "terrace" of the $\mathrm{Cu}(443)$ surface, respectively. Adsorption heights and tilt angles for several $\mathrm{Bz} / \mathrm{Cu}(443)$ metastable configurations are shown in (c). In (d), we show the TPD spectra for the desorption of $\mathrm{Bz}$ and $\mathrm{Np}$ from $\mathrm{Cu}(111)$ adsorbed at low coverage and $\mathrm{Cu}(443)$ adsorbed at low and high coverages. The TPD spectra are adapted from Ref. [19]. 
$\mathrm{Cu}(443)$ terrace: from 0.8 to $1.1 \mathrm{eV}$ for $\mathrm{Bz}(\mathrm{Bz}-\mathrm{T} 1$ to $\mathrm{Bz}-$ $T 11)$ and from 1.3 to $1.6 \mathrm{eV}$ for $\mathrm{Np}$ (Np-T1 to $\mathrm{Np}-T 9)$, where the notation $T 1$ to $T 11$ denotes the possible adsorption sites for $\mathrm{Bz}$ and $\mathrm{Np}$ on the terrace $(T)$ of $\mathrm{Cu}(443)$; see Fig. 1. The adsorption energies $\left(E_{\text {ads }}\right)$ on the terrace depend on the distance $d$ from the step edge; in fact, $E_{\text {ads }}$ in Figs. 1(a) and 1(b) exhibits a nearly linear behavior with respect to $d$. This linear dependence can be mainly attributed to the stronger vdW attraction near the step edge, since the pure PBE adsorption energies are essentially degenerate for all configurations of $\mathrm{Bz}$ and $\mathrm{Np}$ adsorbed on flat terraces. However, also the variation of vertical adsorption heights and local electric fields close and far from the step contribute to the observed quasilinear adsorption energy on the $\mathrm{Cu}(443)$ terraces. Although some of the calculated metastable states may correspond to saddle points, this fact does not change any of our conclusions. The values of $E_{\text {ads }}$ obtained from $\mathrm{PBE}+$ $\mathrm{vdW}^{\text {surf }}$ calculations are in the range of adsorption enthalpies estimated from TPD desorption peaks [19,29]. In contrast, pure PBE calculations [21] lead to a flat PES, with adsorption on terraces being endothermic.

The equilibrium height and tilt of $\mathrm{Bz}$ are sensitive to the adsorption position. On the terrace the molecules are essentially flat with vertical heights between 2.70 and $2.89 \AA$, while for the adsorption on step edges Bz tilts from $22^{\circ}$ to $30^{\circ}$ relative to the terrace and forms covalent bonds to the $\mathrm{Cu}$ atoms at the step with $\mathrm{C}-\mathrm{Cu}$ distances ranging from 2.27 to $2.78 \AA$ from the edge to the molecule center. For $\mathrm{Np}$, the most stable configurations correspond to the long axis of the molecule aligned parallel to the step edge. The adsorption height variation for different metastable states of $\mathrm{Np} / \mathrm{Cu}(443)$ is much smaller than for $\mathrm{Bz} / \mathrm{Cu}(443)$, its average value being $2.76 \AA$.

At this point, it is worthwhile to compare our calculated $E_{\text {ads }}$ values with the available experimental estimates obtained from TPD desorption peaks. To determine adsorption enthalpies from TPD desorption temperatures, we utilize the Redhead equation [30], which requires the knowledge of desorption prefactors that depend on the difference between the entropy of the adsorbed molecule and its counterpart in the gas phase. The usual prefactor $\nu=10^{13} s^{-1}$, derived for desorption of small molecules, was frequently utilized due to the lack of knowledge of precise entropy differences. The situation has been significantly improved after the seminal work of Campbell and Sellers [31,32], who have assembled an extensive data set of $\Delta S$ values for adsorbed molecules. We recently used the Campbell-Sellers method to determine a prefactor of $10^{15.2} \mathrm{~s}^{-1}$ for the desorption of $\mathrm{Bz}$ on coinage metal surfaces [13], which we use here for both $\mathrm{Bz}$ and $\mathrm{Np}$. In short, the Redhead equation can be written as

$$
H_{d}=k_{B} T_{d}\left[\ln \frac{T_{d} \nu}{\beta}-3.64\right],
$$

where $T_{d}$ is the desorption temperature, $k_{B}$ is the Boltzmann constant, $\beta$ is the heating rate, and $\nu$ is the preexponential factor. The adsorption enthalpies determined in this way were converted into energies by adding $3 k_{B} T_{d} / 2$ [13]. Starting with the simplest case of $\mathrm{Bz}$ on

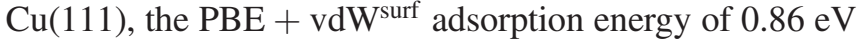
is slightly overestimated compared to $E_{\mathrm{ads}}=0.71 \mathrm{eV}$ determined from the experimental TPD analysis. Since our main interest is investigating the behavior of molecules on $\mathrm{Cu}(111)$ vs $\mathrm{Cu}(443)$, this slight overestimation largely cancels out in energy differences as discussed below.

The adsorption of $\mathrm{Bz}$ and $\mathrm{Np}$ on $\mathrm{Cu}(443)$ and $\mathrm{Cu}(111)$ surfaces has been thoroughly studied experimentally by Lukas et al. [19]. For adsorption at low coverages, they observed an increase of $50 \mathrm{~K}$ in the desorption temperature for $\mathrm{Bz} / \mathrm{Cu}(443)$ when compared to $\mathrm{Bz} / \mathrm{Cu}(111)$ [see Fig. 1(d)]. This difference was attributed to the adsorption of $\mathrm{Bz}$ on step edges of the $\mathrm{Cu}(443)$ surface. Until now, it was not possible to rationalize this observation using firstprinciples calculations due to recent developments of reliable methods for treating vdW interactions for molecules at surfaces. The increase of the desorption temperature from $228 \mathrm{~K}$ for $\mathrm{Bz} / \mathrm{Cu}(111)$ to $278 \mathrm{~K}$ for $\mathrm{Bz} / \mathrm{Cu}(443)$ corresponds to a difference in adsorption energy of $0.15 \mathrm{eV}$. This is a factor of 3 smaller than the difference in adsorption energy of $0.45 \mathrm{eV}$ predicted by our calculations, corresponding to adsorption of $\mathrm{Bz}$ on step edges of $\mathrm{Cu}(443)$ vs terraces on $\mathrm{Cu}(111)$. However, such a comparison assumes that the molecules remain in their ground state geometric configuration close to desorption temperatures, thereby ignoring any kinetic effects.

In the case of $\mathrm{Np} / \mathrm{Cu}(443)$ vs $\mathrm{Np} / \mathrm{Cu}(111)$, the difference between experiments and our calculations is apparently even larger. TPD experiments observe a desorption temperature of $349 \mathrm{~K}$ for $\mathrm{Np} / \mathrm{Cu}(111)$ and $410 \mathrm{~K}$ for $\mathrm{Np} / \mathrm{Cu}(443)$. This amounts to a difference in adsorption energy of $0.2 \mathrm{eV}$, compared to $0.6 \mathrm{eV}$ found in our $\mathrm{PBE}+\mathrm{vdW}^{\text {surf }}$ calculations.

To rationalize this apparently significant discrepancy between TPD experiments and first-principles results, we systematically calculated the migration barriers for diffusion of $\mathrm{Bz}$ and $\mathrm{Np}$ on the $\mathrm{Cu}(443)$ surface for different initial and final metastable states. The energetics of some representative diffusion pathways is shown in Fig. 2. An important observation is that all of the computed barriers are much smaller than the corresponding adsorption energies. This suggests that the adsorbed molecules are likely to be mobile on the $\mathrm{Cu}(443)$ terraces already well below desorption temperatures. TPD experiments can only provide information about the molecules upon desorption, given the macroscopic temperature increase rates. Therefore, the TPD spectra do not contain information about the dynamics of adsorbed molecules prior to the desorption process.

The lowest barrier for diffusion from step edges to terraces of the $\mathrm{Cu}(443)$ surface determined in our 


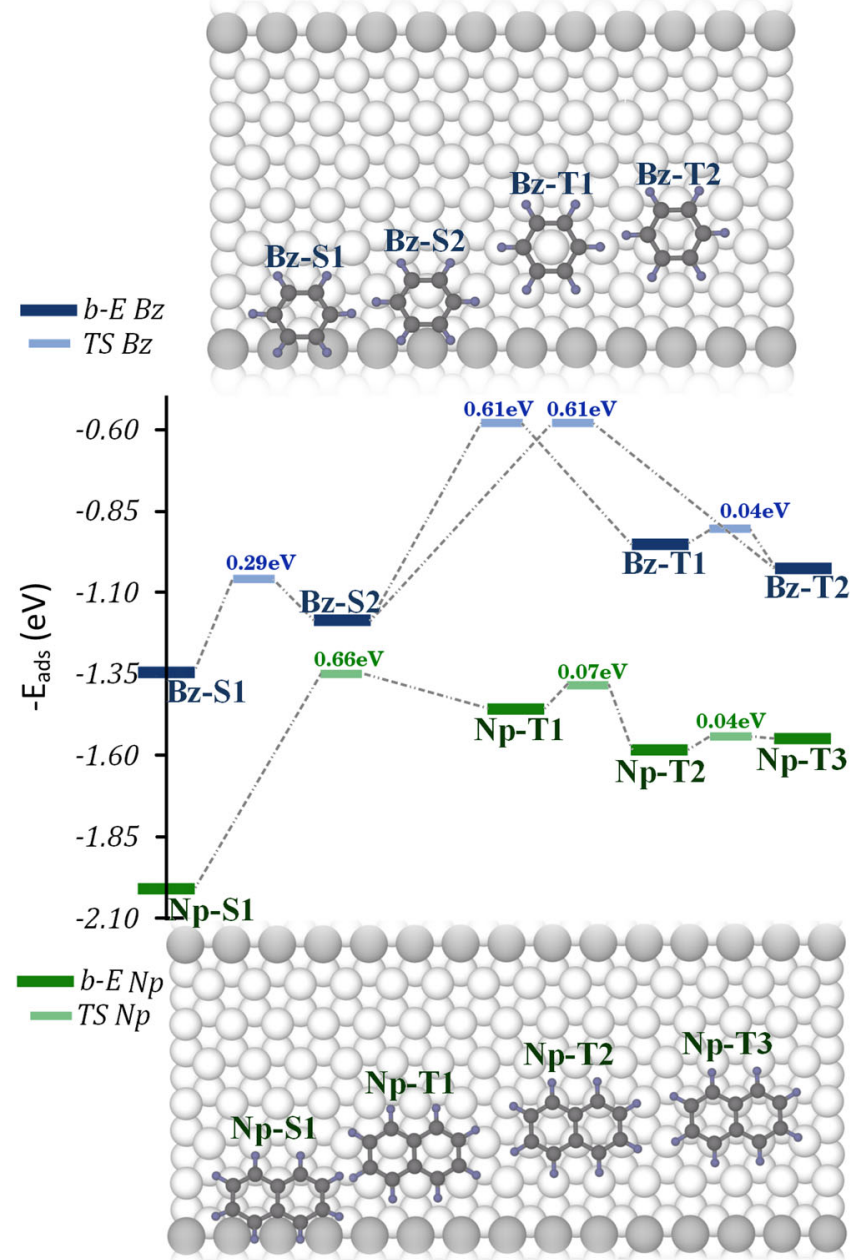

FIG. 2 (color online). Adsorption energies $(b-E)$ and diffusion barriers $(T S)$ for $\mathrm{Bz}$ (upper blue line) and $\mathrm{Np}$ (lower green line), determined from PBE + vdW ${ }^{\text {surf }}$ calculations. Structural models are also shown, where all stable configurations correspond to Fig. 1, viewed from the top.

$\mathrm{PBE}+\mathrm{vdW}$ surf calculations is $0.61 \mathrm{eV}$ for $\mathrm{Bz}$ and $0.66 \mathrm{eV}$ for Np. Using a prefactor of $10^{16} s^{-1}$ in the Arrhenius equation, these barriers would correspond to $\sim 200 \mathrm{~K}$ for $\mathrm{Bz} / \mathrm{Cu}(443)$ and $\sim 220 \mathrm{~K}$ for $\mathrm{Np} / \mathrm{Cu}(443)$. Both temperatures are smaller than the desorption temperatures observed in TPD experiments, thereby providing a strong support for our alternative dynamic explanation of the ADD mechanism for $\mathrm{Bz}$ and $\mathrm{Np}$ on the $\mathrm{Cu}(443)$ surface. Another support for our theoretical predictions is the fact that no ordered diffraction pattern could be observed for $\mathrm{Np} / \mathrm{Cu}(443)$ by LEED at temperatures slightly above $300 \mathrm{~K}$ [19]. This strongly suggests that $\mathrm{Np}$ molecules freely diffuse on the $\mathrm{Cu}(443)$ terraces well below their desorption temperature of $410 \mathrm{~K}$. We note in passing that our conclusions about the migration mechanism would not be modified by changes of the prefactor by 2 orders of magnitude. In addition, we have carried out calculations using a nonlocal vdW functional optB86b-vdW [33], fully reproducing the $\mathrm{PBE}+\mathrm{vdW}^{\text {surf }}$ results.

How then can we explain the increase of $\sim 50 \mathrm{~K}$ in desorption temperature observed in TPD for $\mathrm{Cu}(443)$ compared to $\mathrm{Cu}(111)$ ? Since the migration barriers between different metastable states on $\mathrm{Cu}(443)$ terraces are significantly below the desorption temperature, we can simply use a Boltzmann average over the energies of all accessible metastable states to calculate the adsorption energy on $\mathrm{Cu}(443)$ at a given desorption temperature. Using this procedure, we obtain an increase of $0.16 \mathrm{eV}$ for the adsorption on terraces of $\mathrm{Bz} / \mathrm{Cu}(443)$ compared to $\mathrm{Bz} / \mathrm{Cu}(111)$, while for $\mathrm{Np} / \mathrm{Cu}(443)$ the corresponding increase is $0.19 \mathrm{eV}$. These values are in excellent agreement with the peaks observed in TPD experiments that lead to an increase of the adsorption energy of $0.15 \mathrm{eV}$ when going from $\mathrm{Bz} / \mathrm{Cu}(111)$ to $\mathrm{Bz} / \mathrm{Cu}(443)$ and to an increase of $0.20 \mathrm{eV}$ when going from $\mathrm{Np} / \mathrm{Cu}(111)$ to $\mathrm{Np} / \mathrm{Cu}(443)$. This analysis demonstrates that the desorption signal from $\mathrm{Cu}(443)$ arises from mobile molecules adsorbed on the terraces of the $\mathrm{Cu}(443)$ surface and not from molecules located at step edges.

Finally, we rationalize experimental observations for the adsorption of $\mathrm{Bz}$ and $\mathrm{Np}$ on $\mathrm{Cu}(443)$ for monolayer and higher coverages, where the desorption mechanism is even more complex. In this case, two well-resolved peaks appear in the TPD spectra [see Fig. 1(d)]: for $\mathrm{Bz} / \mathrm{Cu}(443)$ at 190 and $260 \mathrm{~K}$, while for $\mathrm{Np} / \mathrm{Cu}(443)$ the peaks are located at 300 and $385 \mathrm{~K}$. The coexistence of two peaks was interpreted as an indication of initial desorption from terraces, followed by the desorption from step edges. It is noticeable that the temperature of the higher desorption peak remains similar as in the case of adsorption on $\mathrm{Cu}(443)$ at low coverages, while the lower peak is displaced to decreasing temperatures even when compared to desorption from $\mathrm{Cu}(111)$. In experiments, it is evident that both step edges and terraces are populated with $\mathrm{Bz}$ and Np molecules upon increasing the surface coverage [19]. Since adsorption on terraces leads to lower stability, these molecules desorb first, explaining the first TPD peak at $190 \mathrm{~K}$ for $\mathrm{Bz} / \mathrm{Cu}(443)$ and $300 \mathrm{~K}$ for $\mathrm{Np} / \mathrm{Cu}(443)$. The low temperatures of these peaks arise from repulsive lateral $\mathrm{Bz}-\mathrm{Bz}$ (Np-Np) interactions at monolayer and higher coverages. Our extensive calculations for full monolayers and double layers (with up to six molecules per $4 \times 1$ unit cell for $\mathrm{Bz}$ and $6 \times 1$ unit cell for $\mathrm{Np}$ ) yield adsorption energies for the "weakest" molecules of $0.65 \pm 0.1 \mathrm{eV}$ for $\mathrm{Bz}$ and $0.96 \pm 0.1 \mathrm{eV}$ for $\mathrm{Np}$. These values agree particularly well with the adsorption energies of $0.59 \mathrm{eV}$ $\left(T_{d}=190 \mathrm{~K}\right)$ for $\mathrm{Bz}$ and $0.94 \mathrm{eV}\left(T_{d}=300 \mathrm{~K}\right)$ for $\mathrm{Np}$, determined using Eq. (1) from experimental lowtemperature desorption peaks. Once some of the terrace molecules desorb, the temperature is already sufficiently high for the molecules on the step edges to overcome the migration barrier and diffuse into the terraces. We note that 
this process can be rather cooperative, as suggested by the very broad nature of the high temperature peak in TPD experiments for high-coverage $\mathrm{Bz}$ and $\mathrm{Np}$ layers on $\mathrm{Cu}(443)$, as shown in Fig. 1(d) [19]. Upon migration of the "former step-edge" molecules onto $\mathrm{Cu}(443)$ terraces, the lateral repulsion is now reduced, leading to a higher adsorption strength compared to monolayer coverage and explaining the high-temperature peaks observed in TPD experiments for both $\mathrm{Bz}$ and $\mathrm{Np}$. Our analysis also explains why high-temperature or high-coverage peaks arise at temperatures similar to desorption at low coverages. Once the former step-edge molecules have diffused onto $\mathrm{Cu}(443)$ terraces, their adsorption energy becomes identical to the scenario where the molecules are adsorbed at low coverage.

In conclusion, we have studied the adsorption, diffusion, and desorption behavior of benzene and naphthalene molecules on the vicinal $\mathrm{Cu}(443)$ surface. Our calculations suggest a more complex dynamical picture than previously proposed from temperature-programed desorption studies of these systems. In general, our findings imply that the understanding of molecular adsorption at realistic surfaces at finite temperatures requires a consideration of dynamical effects. This strongly advocates the need for time-resolved experimental studies to understand the behavior of molecular layers on realistic surfaces.

The authors acknowledge support from the European Research Council (ERC Starting Grant VDW-CMAT). W. L. acknowledges support from the National Natural Science Foundation of China (No. 21403113) and the Fundamental Research Funds for the Central Universities (No. 30915011330).

J. C.-C. and W. L. contributed equally to this work.

*tkatchenko@fhi-berlin.mpg.de

[1] N. Koch, N. Ueno, and A. T. S. Wee, The Molecule-Metal Interface (Wiley, New York, 2013).

[2] L. Zoppi, L. Martin-Samos, and K. K. Baldridge, Acc. Chem. Res. 47, 3310 (2014).

[3] K. Golibrzuch, P. R. Shirhatti, J. Geweke, J. Werdecker, A. Kandratsenka, D. J. Auerbach, A. M. Wodtke, and C. Bartels, J. Am. Chem. Soc. 137, 1465 (2015).

[4] E. Goiri, M. Matena, A. El-Sayed, J. Lobo-Checa, P. Borghetti, C. Rogero, B. Detlefs, J. Duvernay, J. Ortega, and D. de Oteyza, Phys. Rev. Lett. 112, 117602 (2014).

[5] T. K. Haxton, H. Zhou, I. Tamblyn, D. Eom, Z. Hu, J. B. Neaton, T. F. Heinz, and S. Whitelam, Phys. Rev. Lett. 111, 265701 (2013).
[6] K. Forster-Tonigold, X. Stammer, C. Wöll, and A. Groß, Phys. Rev. Lett. 111, 086102 (2013).

[7] F. S. Tautz, Prog. Surf. Sci. 82, 479 (2007).

[8] L. Kronik and N. Koch, MRS Bull. 35, 417 (2010).

[9] T. Roman and A. Groß, Phys. Rev. Lett. 110, 156804 (2013).

[10] J. K. Nørskov, F. Abild-Pedersen, F. Studt, and T. Bligaard, Proc. Natl. Acad. Sci. U.S.A. 108, 937 (2011).

[11] W. Liu, A. Tkatchenko, and M. Scheffler, Acc. Chem. Res. 47, 3369 (2014).

[12] W. Liu, J. Carrasco, B. Santra, A. Michaelides, M. Scheffler, and A. Tkatchenko, Phys. Rev. B 86, 245405 (2012).

[13] W. Liu, V. G. Ruiz, G.-X. Zhang, B. Santra, X. Ren, M. Scheffler, and A. Tkatchenko, New J. Phys. 15, 053046 (2013).

[14] L. Bartels, Nat. Chem. 2, 87 (2010).

[15] C. Sanchez, P. Belleville, M. Popall, and L. Nicole, Chem. Soc. Rev. 40, 696 (2011).

[16] J. D. Horvath, A. Koritnik, P. Kamakoti, D. S. Sholl, and A. J. Gellman, J. Am. Chem. Soc. 126, 14988 (2004).

[17] R. Zhang et al., Phys. Chem. Chem. Phys. 15, 20662 (2013).

[18] S. González, F. Viñes, J. F. García, Y. Erazo, and F. Illas, Surf. Sci. 625, 64 (2014).

[19] S. Lukas, S. Vollmer, G. Witte, and C. Wöll, J. Chem. Phys. 114, 10123 (2001).

[20] V. G. Ruiz, W. Liu, E. Zojer, M. Scheffler, and A. Tkatchenko, Phys. Rev. Lett. 108, 146103 (2012).

[21] J. Perdew, K. Burke, and M. Ernzerhof, Phys. Rev. Lett. 77, 3865 (1996).

[22] W. A. Al-Saidi, H. Feng, and K. A. Fichthorn, Nano Lett. 12, 997 (2012).

[23] C. Wagner, N. Fournier, F. S. Tautz, and R. Temirov, Phys. Rev. Lett. 109, 076102 (2012).

[24] V. Blum, R. Gehrke, F. Hanke, P. Havu, V. Havu, X. Ren, K. Reuter, and M. Scheffler, Comput. Phys. Commun. 180, 2175 (2009).

[25] N. Govind, M. Petersen, G. Fitzgerald, D. King-Smith, and J. Andzelm, Comput. Mater. Sci. 28, 250 (2003).

[26] S. J. Clark, M. D. Segall, C. J. Pickard, P. J. Hasnip, M. I. Probert, K. Refson, and M. C. Payne, Z. Kristallogr. 220, 567 (2005).

[27] See Supplemental Material at http://link.aps.org/ supplemental/10.1103/PhysRevLett.115.086101 for more details regarding calculations and analysis.

[28] S. Stranick, M. Kamna, and P. Weiss, Surf. Sci. 338, 41 (1995).

[29] M. Xi, M. Yang, S. Jo, B. Bent, and P. Stevens, J. Chem. Phys. 101, 9122 (1994).

[30] P. A. Redhead, Vacuum 12, 203 (1962).

[31] C. T. Campbell and J. R. V. Sellers, J. Am. Chem. Soc. 134, 18109 (2012).

[32] C. T. Campbell and J. R. V. Sellers, Chem. Rev. 113, 4106 (2013).

[33] J. Klimeš, D. R. Bowler, and A. Michaelides, J. Phys. Condens. Matter 22, 022201 (2010). 